in vivo $33: 419-424(2019)$

doi:10.21873/invivo.11489

\title{
Osteogenic Evaluation of Goose-beak Bones According to Processing Temperature
}

\author{
JONG MIN KIM ${ }^{1}$ and SEOK HWA CHOI ${ }^{2}$ \\ ${ }^{1}$ Xenotransplantation Research Center, Seoul National University, Seoul, Republic of Korea; \\ ${ }^{2}$ Department of Veterinary Surgery, College of Veterinary Medicine, \\ Chungbuk National University, Cheongju, Republic of Korea
}

\begin{abstract}
Background/Aim: As an alternative material to autogenous bone, goose-beak bone particles (GBP) have been attracting great attention as a bone substitute due to their biological properties. This study was performed to assess bone generation using GBP in calvarial defects in a rat model. The study focus was the osteogenic potential of goose-beak bone at different processing temperatures. Materials and Methods: There were three experimental groups: Control group (critical defect only), low-temperature (LT) group (filled with GBP heat-treated for 20 h at $400^{\circ} \mathrm{C}$ ), and high-temperature (HT) group (filled with GBP heattreated for $3 \mathrm{~h}$ at $\left.1,200^{\circ} \mathrm{C}\right)$. Results: The Ca/P atomic ratio of the goose-beak bone was 1.63, and the bones had a bony structure with open pores and interconnected rod-like struts. Micro-computed tomographic analysis revealed the quantity of new bone formation of the HT group was higher than that of the LT group. At 12 weeks after GBP insertion, new bone formation was significantly higher $(p<0.05)$ in the HT group, and there were more new osteocytes in the lacuna in the HT group than in the LT group. Thus, GBP treated at a high temperature formed more new bone than that treated at a low temperature. Conclusion: It can be concluded that HTtreated GBP is a graft material that can be effective in promoting bone formation.
\end{abstract}

Many cases of bone damage are due to trauma, bone tumors, and metabolic diseases. For the repair of bone damage, a bone substitute may be used (1). Autograft bone material is

This article is freely accessible online.

Correspondence to: Professor Seok Hwa Choi, DVM, Ph.D., Department of Veterinary Surgery, College of Veterinary Medicine, Chungbuk National University, Cheongju 28644, Republic of Korea. Tel: +82 432613144, Fax: +82 432613224, e-mail: shchoi@cbu.ac.kr

Key Words: Goose beak, bone defect, osteogenesis, temperature, rat. an ideal solution for use in bone grafting as it has the benefit of osteogenic activity. However, the supply of autograft material is limited (2). Xenograft materials are common substitutes for autografts. Xenograft materials are widely used today in dental surgery, orthopedics, and plastic surgery. For the treatment or regeneration of damaged human bone, xenografts composed of ceramic materials derived from xenogeneic bone such as bovine or porcine bone are frequently utilized (2). However, xenografts have disadvantages, including immune rejection, transmission of animal disease into humans, and supply limitation (3). Of particular concern was the use of xenografts based on bovine bone material as is raises the possibility of a transfer of bovine spongiform encephalopathy (3).

Domestic geese are mainly used for meat, eggs, and feather production, and their oval-shaped heads are usually discarded or used to produce natural organic fertilizers and animal feeds. The goose beak is composed of keratin and comprises two major bones in each side of the lower and upper bill. As farming of domestic geese could become a potential source of domestic goose beaks, and as geese have a relatively low chance of harboring a zoonose, goose beakderived xenografts may be a more suitable xenograft material for bone grafting.

Biomedical ceramics have been used for treating bone loss or repairing bone damage due to their superior mechanical properties and biocompatibility compared to polymer materials. However, the use of synthetic bone grafts or bone xenograft materials provides another option to the use of autografts $(4,5)$. Human bone apatite has a low crystallinity; however, most commercially available artificial bone substitutes have extremely crystalline properties due to the high temperatures used during their production. For example, hydroxyapatite (HA) is very crystalline because of the sintering procedure used during its production, and it has a larger particle size than bone apatite due to grain expansion. Such large particles are extremely resistant to biodegradation in the body; moreover, their osteoconduction is notably low and they cannot be degraded by osteoclasts (6). Based on 
those findings, it is thought that both heating temperature and crystallinity of graft materials may have effects on the bone healing area adjacent to bone graft particles. Thus, in this study, we compared the osteogenic potential of goose-beak bone particles (GBP) processed at low and high temperatures in healing of a critical-sized defect in a rat model.

\section{Materials and Methods}

Preparation of GBP. In order to collect suitable stock material of goose-beak bone tissue from livestock geese (Emden breed, males, 17 weeks old), all soft tissues were removed from goose beaks and associated bone surgically extracted from harvested geese. The harvested bone samples were thoroughly washed to remove macroscopically adhered impurities and boiled in distilled water for $8 \mathrm{~h}$. Next, the washed samples were deproteinized with hydrogen dioxide for $8 \mathrm{~h}$ (7). The deproteinized bone samples were then dried for $48 \mathrm{~h}$ in an oven at $60^{\circ} \mathrm{C}$. The goose-beak bone samples were cut into pieces $\leq 2 \mathrm{~cm}^{3}$. To withdraw any remaining blood and hydrogen dioxide, the goose-beak bones were rinsed with distilled water. Subsequently, half to the samples were sintered for $20 \mathrm{~h}$ at $400^{\circ} \mathrm{C}$ and the other half sintered for $3 \mathrm{~h}$ at $1,200^{\circ} \mathrm{C}$ in an electronic furnace (MF-21G; JeioTech, Daejon, Korea). Through the above processes, all organic matrices and disease-causing agents were removed from the samples. For use, the sintered goose-beak bone pieces were milled into 500-700 $\mu \mathrm{m}$ particles. To remove moisture residues totally, the milled bone particles were freeze-dried, then maintained at room temperature.

Morphological analyses of GBP. Analysis of the morphological features of sintered GBP was performed by using a scanning electron microscope (SEM, S-4300; Hitachi Co., Hitachi, Japan). Before examination, samples were gold sputter-coated to render them electrically conductive. The calcium-phosphorus ratio of GBP was analyzed by using an energy dispersive spectrometer. The obtained profiles were analyzed with a field emission gun SEM system (Quanta 200 FEG; FEI Co., Hillsboro, OR, USA) at a voltage of $15 \mathrm{kV}$, spot size 3 , and a random 5 point.

In vivo study. Twenty healthy 8-week-old male Sprague-Dawley rats (Samtaco, Osan, Korea; weight=250-280 g) were used. The rats were housed in an air-conditioned room with a $12 \mathrm{~h} \mathrm{light/dark} \mathrm{cycle} \mathrm{and}$ controlled temperature $\left(23 \pm 2^{\circ} \mathrm{C}\right)$ and relative humidity $(60 \% \pm 10 \%)$. The animals were divided into three groups: Control group: untreated critical defect $(\mathrm{n}=10)$; low-temperature (LT) group: defect filled with GBP heat-treated for $20 \mathrm{~h}$ at $400^{\circ} \mathrm{C}(\mathrm{n}=5)$; and high-temperature (HT) group: defect filled with GBP heat-treated for $3 \mathrm{~h}$ at $1,200^{\circ} \mathrm{C}$ $(n=5)$. Animal experimentation procedures were approved by the Institutional Animal Care and Use Committee of Chungbuk National University (Approval CA-16-21). Prior to operation, rats were given complete anesthesia by intraperitoneal injection of $40 \mathrm{mg} / \mathrm{kg}$ ketamine (Ketamine50 ${ }^{\circledR}$; Yuhan Co., Seoul, Korea) and $10 \mathrm{mg} / \mathrm{kg}$ xylazine (Rompun ${ }^{\circledR}$; Bayer Korea, Seoul, Korea). Both sides of the calvaria of rats were drilled to create a $6 \mathrm{~mm}$ hole, after which heattreated GBP were inserted, as appropriate. Twelve weeks after particle insertion, calvarial tissue at the defect area was harvested and fixed with $10 \%$ neutral buffered formalin. The presence of osteogenesis was determined via micro-computed tomography (CT) and histological examination.
Micro-CT analysis. Fixed calvaria specimens were imaged using an in vivo high-resolution micro-CT system (Skyscan 1176; Skyscan, Aartselaar, Belgium). The samples were scanned using a pixel size of $12.56 \mu \mathrm{m}$, a $1 \mathrm{~mm}$ aluminum filter, a rotation step of $0.5^{\circ}$ and a rotation angle of $180^{\circ}$. The $\mathrm{X}$-ray tube voltage was $65 \mathrm{kV}$ and the current was $385 \mu \mathrm{A}$. Raw images were reconstructed by using NRecon Skyscan reconstruction software (Skyscan). Reconstructed images were used to determine that percentage of new bone formation and to create two-dimensional (2D) and three-dimensional (3D) images.

Histological evaluation. After scanning to obtain micro-CT images, calvarial tissues around the defect area were harvested and fixed in a $10 \%$ formalin solution for $24 \mathrm{~h}$ at room temperature. Subsequently, the samples were dehydrated in an ascending series of alcohol rinses. Dehydrated samples were then embedded in acrylic resin (Technovit 7200 VLC; Kulzer \& Co GmbH, Friedrichdorf, Germany) and serially cut into $20 \mu \mathrm{m}$-thick sections. The section specimens were mounted on glass slides and stained with hematoxylin and eosin (H\&E) and Masson's trichrome (MT). The stained tissue samples were observed under an optical microscope.

Statistical analysis. The results are expressed as mean \pm standard deviation values. For determining the statistical significance of differences between two groups, results were analyzed by one-way analysis of variance followed by Tukey's test. Differences with $p<0.05$ were considered statistically significant.

\section{Results}

Morphological characteristics of GBP. Goose beaks used in this study consisted mostly of bone covered by a thin dermis (Figure 1A). The average length of the upper beak bone was $13.4 \mathrm{~cm}$, while that of the lower bone was $7.6 \mathrm{~cm}$. SEM images of goose-beak bones revealed a bony structure with open pores and interconnected rod-like struts. Prepared GBP in the HT group had many pores in the macro- and micrometer size range. The HT particle had a porosity of $77.37 \%$ and a pore size of $2.78 \mu \mathrm{m}$ (Figure 1B). The characteristics of the GBP in the LT group were broadly similar to those of the HT group. The $\mathrm{Ca} / \mathrm{P}$ atomic ratio in the HT and LT groups was 1.63 and analysis did not reveal any distinguishable crystalline impurities (Figure 2).

Micro-CT analyses of new bone formation. In vivo quantitative analyses of total new bone volume (including GBP and newly formed bone volume), and newly formed bone volume (excluding GBP) by thresholding were undertaken by examining micro-CT images. The percentage of newly formed bone volume out of total new bone volume in the HT and LT groups was $26.27 \% \pm 6.67 \%$ and $17.30 \% \pm 5.36 \%$, respectively (Figure 3 ). The volume of newly-formed bone in the HT group indicated that the HTtreated particles provided significantly higher osteogenic potential than that of the LT-treated particles $(p<0.05)$. 


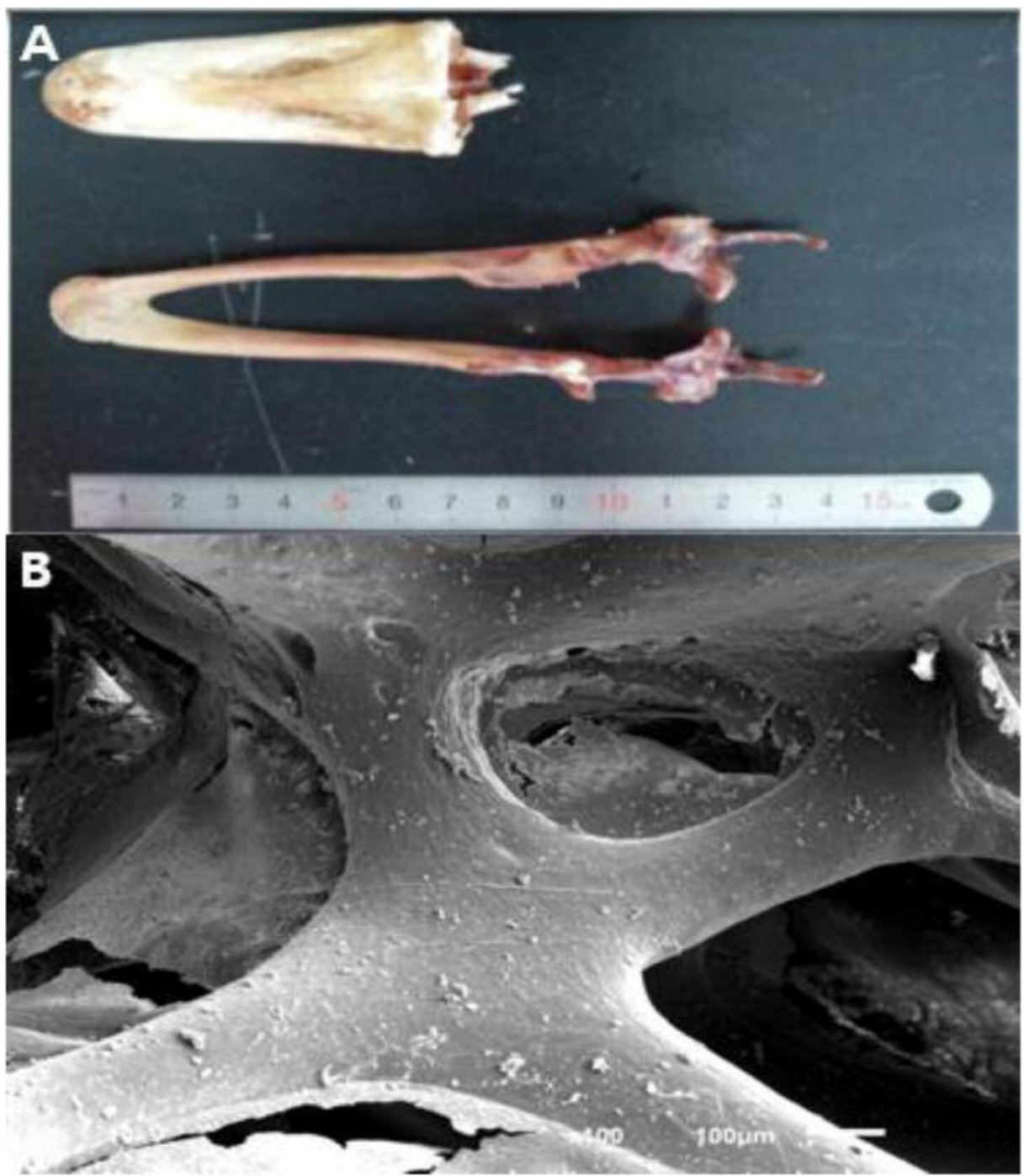

Figure 1. Gross view (A) and scanning electron microscopy image (B) of a goose-beak bone.

Histological evaluation of bone regeneration. Microscopic images obtained after H\&E and MT staining of crosssectional $2 \mathrm{D}$ and maximum intensity projection micro-CT images were compared (Figure 3). The color-mapped, maximum intensity projection images helped visualize the mineral density of the new bone. Viewed under confocal microscopy, yellow (oxytetracycline), green (calcein), and red (alizarin) staining of sections indicated the presence of new bone formation. Typical H\&E and MT staining of nondecalcified sections was also observed. Examination indicated that osteogenesis was initiated from the periphery of the host bone and showed that the newly formed bone was well integrated with the GBP in both the HT and LT groups. Remarkable bone apposition was observed at the edge of the interface of the host bone with GBP. Importantly, we observed a number of osteoblasts and osteoclasts in both groups (Figure 4).

\section{Discussion}

Biomedical ceramics, because of their superior mechanical properties and biocompatibility compared to the polymer materials, are widely used today in various medical fields such as dental surgery, orthopedics, and plastic surgery for treating or repairing bone loss or damage $(3,8)$. Ceramics of calcium phosphate, bioactive glass, alumina, zirconia, and complexes thereof are widely utilized in biomedical applications. Calcium phosphate ceramics include HA, tricalcium phosphate (TCP), tetra-calcium phosphate, and dicalcium phosphate, whilst bioactive glass materials include 


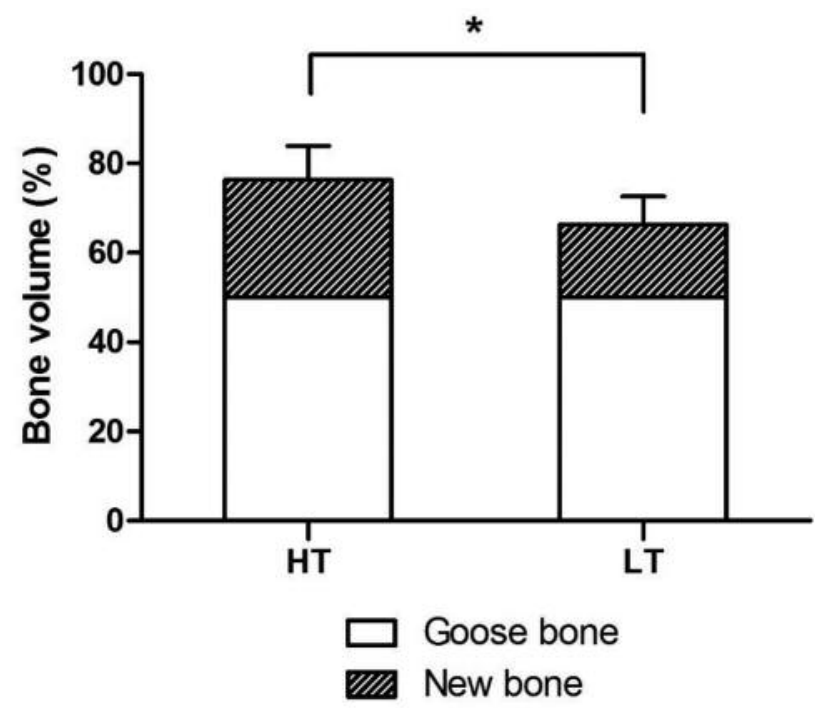

Figure 2. The result of energy dispersive spectrometric analysis of particles derived from goose-beak bone.

silica-based glass, phosphate-based glass, and glass ceramics (9). Recently, many researchers have attempted to extract HA from natural resources such as corals, seashells, egg shells, cuttlefish shells, natural gypsum, and natural calcite (3). These raw materials are mainly composed of $\mathrm{CaCO}_{3}$ or $\mathrm{CaSO}_{4}$, and an additional chemical reaction is necessary to produce HA. In contrast goose-beak bone is a natural bioderived HA that has important advantages: Goose-beak bones are available at a very low material cost as a byproduct of goose farming, and they can be processed easily and quickly. Regardless of this, as far as we are aware, there are no reports describing the development and use of bioceramic goose beak-derived bone.

Bone composition ratios vary depending on the processing temperature of the bone (10). Moreover, even if the same material is used, its crystalline structure can change depending on the treatment temperature (2). Crystalline human bone has been shown to vary under various sintering conditions, and its degree of crystallinity increases as the heating temperature increase (11). Porcine cancellous bone particles had larger surface area and greater pore volume after low-temperature treatment $\left(5 \mathrm{~h}\right.$ at $\left.400^{\circ} \mathrm{C}\right)$ than after high-temperature treatment $\left(3 \mathrm{~h}\right.$ at $\left.1,200^{\circ} \mathrm{C}\right)(2)$. In addition, the densification of apatite particles in porcine cancellous bone particle was increased by a high-temperature treatment (2). However, crystallinity of ovine cortical bone increased with increased heating temperature and processing time (12). A material with a suitable composition ratio and an excellent possibility of being incorporated via osteogenesis is HA treated at $1,200^{\circ} \mathrm{C}(13)$.

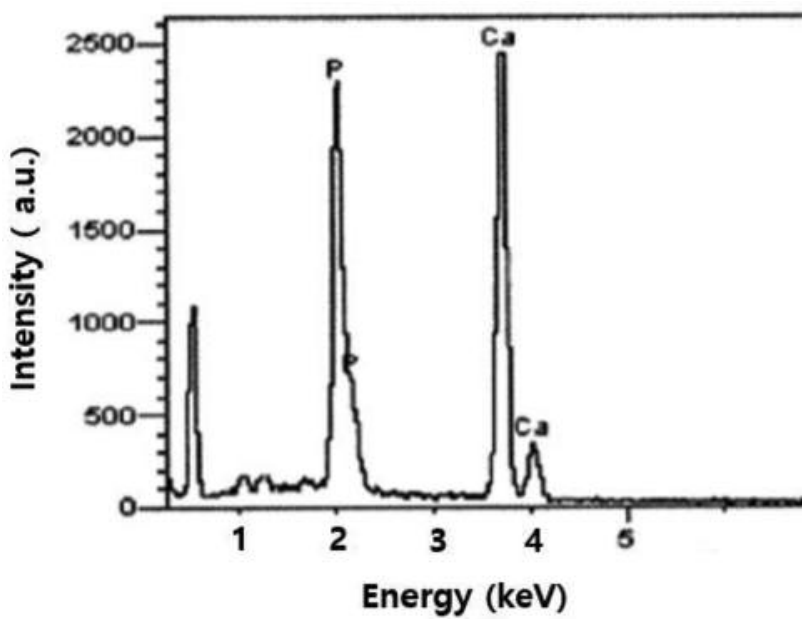

Figure 3. The average percentage of new bone formation at 12 weeks after insertion of goose-beak bone particles. HT: Heated at $1,200^{\circ} \mathrm{C}$ for $3 \mathrm{~h}$; LT: heated at $400^{\circ} \mathrm{C}$ for $20 \mathrm{~h}$. *Significantly different at $p<0.05$.

The most widely used calcium phosphate ceramic is HA whose molar ratio of calcium to phosphorus ranges from 1 . 2 to almost 2, which is very similar to that of human bone $(\mathrm{Ca} / \mathrm{P}$ ratio=1.50-1.70). In this study, we attempted to determine the optimum bone processing temperature for preparing GBP that had been deproteinized with hydrogen dioxide. The atomic $\mathrm{Ca} / \mathrm{P}$ ratio of GBP was 1.63 and our analysis did not reveal any distinguishable crystalline impurities. GBP were identified as HA with high crystallinity resulting from the heating process. The crystallized GBP had a high surface area, large pore size, and high pore volume. Such structural characteristics are very important for osteoblast and bone regeneration (14). Our micro-CT evaluations revealed that high-temperature processing had a higher osteogenic effect than did lowtemperature processing. The HT group showed a higher level of new bone formation originating from the defect margin and a good level of bone regeneration. These results further indicate that the HT treatment produced material with better osteoconduction, and osteoinduction than that after LT treatment. In addition, the results show that GBP can be used as an autograft substitute. Porcine bones, like those in other mammals, are hard, but goose bones are porous; thus, processing of goose-beak bone is easier than that of pigs. In addition, we found heat treatment for a short time at a high temperature to be effective for goose-beak bone processing.

Bone regeneration was confirmed by histological examination. When bone regeneration started, osteoblasts formed and the osteoblasts then became osteocytes and 


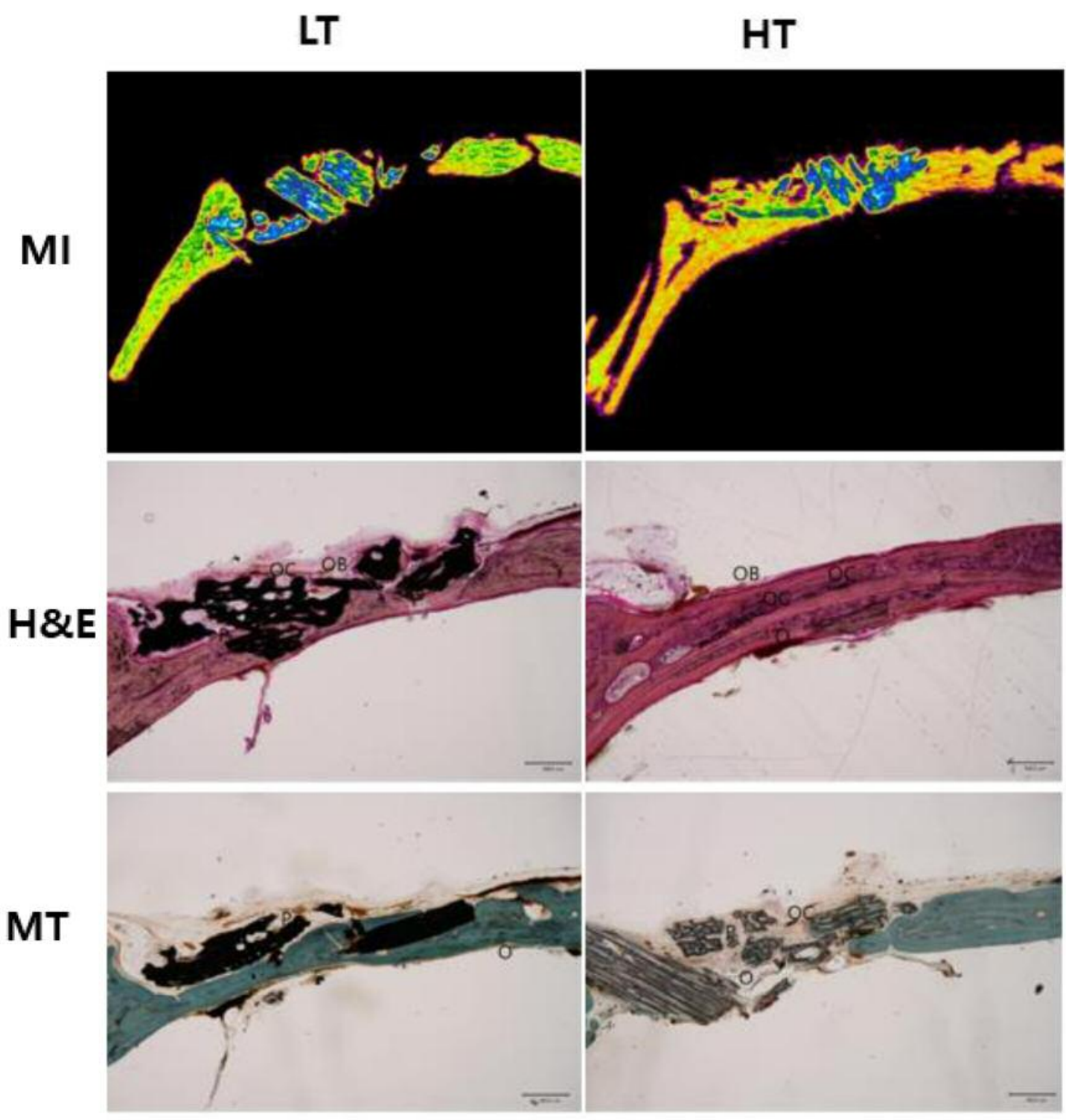

Figure 4. Cross sectional micro-computed tomographic images at 12 weeks post-insertion of goose-beak bone particles in a rat model of critical calvarial defect. Bar $=500 \mu \mathrm{m}$. HT: Heated at 1,200 ${ }^{\circ} \mathrm{C}$ for $3 \mathrm{~h}$; LT: heated at $400^{\circ} \mathrm{C}$ for $20 \mathrm{~h}$. NB: New bone; P: goose-beak bone particles; O: osteocyte; OB: osteoblast; OC: osteoclast.

osteoclasts. The HT group showed better osteogenic effects than did the LT group; however, in both groups, there was new bone formation beginning from the defect margin, and the lacunae of heat-treated bone particles were filled with new osteocytes. As a result of comparing bone formation by processing temperature, the HT group showed significantly improved new bone formation over that in the LT group. Thus, processing goose-beak bone at $1,200^{\circ} \mathrm{C}$ for $3 \mathrm{~h}$ is superior to processing at $400^{\circ}$ for $20 \mathrm{~h}$. Our results are consistent that those in another study showing very good osteogenic potential at $1,200^{\circ} \mathrm{C}(13)$.
As a result, this study confirms that a ceramic powder obtained from goose-beak bones is suitable for bone regeneration applications. Furthermore, once the issue of possible immune system effects has been resolved, it may be possible to develop a superior bone xenograft using goose-beak bone materials.

\section{Acknowledgements}

This research was supported by Basic Science Research Program through the National Research Foundation of Korea (NRF) funded by the Ministry of Education (2018R1D1A1A09083556). 


\section{References}

1 Kim JM, Son JS, Kang SS, Kim G and Choi SH: Bone regeneration of hydroxyapatite/alumina bilayered scaffold with $3 \mathrm{~mm}$ passage-like medullary canal in canine tibia model. BioMed Res Int 2015: 235108, 1-6, 2015. PMID: 25688353. doi.org/10.1155/2015/235108.

2 Go Ara, Kim SE, Shim KM, Lee SM, Choi SH, Son JS and Kang SS: Osteogenic effect of low-temperature-heated porcine bone particles in a rat calvarial defect model. J Biomed Mater Res A 102: 3609-3617, 2014. PMID: 24248774. doi: 10.1002/ jbm.a.35022.

3 Son JS, Jang SH, Kwon TY, Kim KH, Kang SS and Choi SH: Preliminary evaluation of bone graft substitute produced by bone of duck beak. Materials Lett 121: 181-184, 2014. doi: 10.1016/j.matlet.

4 Ilan DI and Ladd AL: Bone graft substitutes. Oper Tech Plast Reconstr Surg 9: 151-160, 2003. doi.org/10.1053/j.otpr.

5 Giannoudis PV, Dinopoulos $\mathrm{H}$ and Tsiridis E: Bone substitutes: An update. Injury 36: S20-S27, 2005. PMID: 16188545. doi: 10.1016/j.injury.

6 Balasundaram G, Sato M and Webster TJ: Using hydroxyapatite nanoparticles and decreased crystallinity to promote osteoblast adhesion similar to functionalizing with RGD. Biomaterials 27: 2798-2805, 2006. PMID: 16430957. doi.org/10.1016/ j.biomaterials.

7 Kim JH, Park MH, Jang SJ, Son SJ, Lee JY, Son JS, Kim SE, Kang SS and Choi SH: Effect of hydrogen dioxide treatment on the osteogenic potential of duck-beak bone-derived natural bioceramic microparticles. In Vivo 31: 373-379, 2017. PMID: 28438865. doi: 10.21873/invivo.11069.

8 Son JS, Appleford M, Ong JL, Wenke JC, Kim JM, Choi SH and Oh DS: Porous hydroxyapatite scaffold with three-dimensional localized drug delivery system using biodegradable microspheres. J Control Release 153: 133-140, 2011. PMID: 21420453. doi.org/10.1016/j.jconrel.
9 Choi SH, Son JS, Kang SS and Lee CH: Production method for biomedical and industrial material using ceramic derived from birds' beaks. US Patent App. 9,855,365 B2, 2018.

10 Miculescu F, Ciocan LT, Miculescu M and Ernuteanu A: Effect of heating process on micro structure level of cortical bone prepared for the compositional analysis. Dig J Nanomater Bios 6: 225-233, 2011. https://www.researchgate.net/publication/ 277650321

11 Rogers KD and Daniels P: An X-ray diffraction study of the effects of heat treatment on bone mineral microstructure. Biomaterials 23: 2577-2585, 2002. PMID: 12033606. doi.org/10.1016/S0142-9612(01)00395-7.

12 Hiller JC, Thompson TJU, Evison MP, Chamberlainc AT and Wessa TJ: Bone mineral change during experimental heating: An X-ray scattering investigation. Biomaterials 24: 5091-5097, 2003. doi.org/10.1016/S0142-9612(03)00427-7.

13 van Gaalen SM, Kruyt MC, Geuze RE, de Bruijn JD, Alblas J and Dhert WJ: Use of fluorochrome labels in in vivo bone tissue engineering research. Tissue Eng Part B: Reviews. 16: 209-217, 2010. PMID: 19857045. doi.org/10.1089/ten.teb.2009.0503

14 Kim JM, Han TS, Kim MH, Oh DS, Kang SS, Kim G, Kwon TY, Kim KH, Lee KB, Son JS and Choi SH: Osteogenic evaluation of calcium phosphate scaffold with drug-loaded poly (lactic-co-glycolic acid) microspheres in Beagle dogs. Tissue Eng Regen Med 9: 175-183, 2012. Doi: 10.1007/s13770-0120175-5. 